This item was submitted to Loughborough's Research Repository by the author.

Items in Figshare are protected by copyright, with all rights reserved, unless otherwise indicated.

\title{
Experimental confirmation of the propulsion of marine vessels employing guided flexural waves in attached elastic fins
}

PLEASE CITE THE PUBLISHED VERSION

http://dx.doi.org/10.1016/j.jfluidstructs.2006.08.015

PUBLISHER

(C) Elsevier

VERSION

AM (Accepted Manuscript)

LICENCE

CC BY-NC-ND 4.0

\section{REPOSITORY RECORD}

Krylov, Victor V., and Gareth V. Pritchard. 2013. "Experimental Confirmation of the Propulsion of Marine Vessels Employing Guided Flexural Waves in Attached Elastic Fins”. figshare.

https://hdl.handle.net/2134/11840. 
This item was submitted to Loughborough's Institutional Repository (https://dspace.lboro.ac.uk/) by the author and is made available under the following Creative Commons Licence conditions.

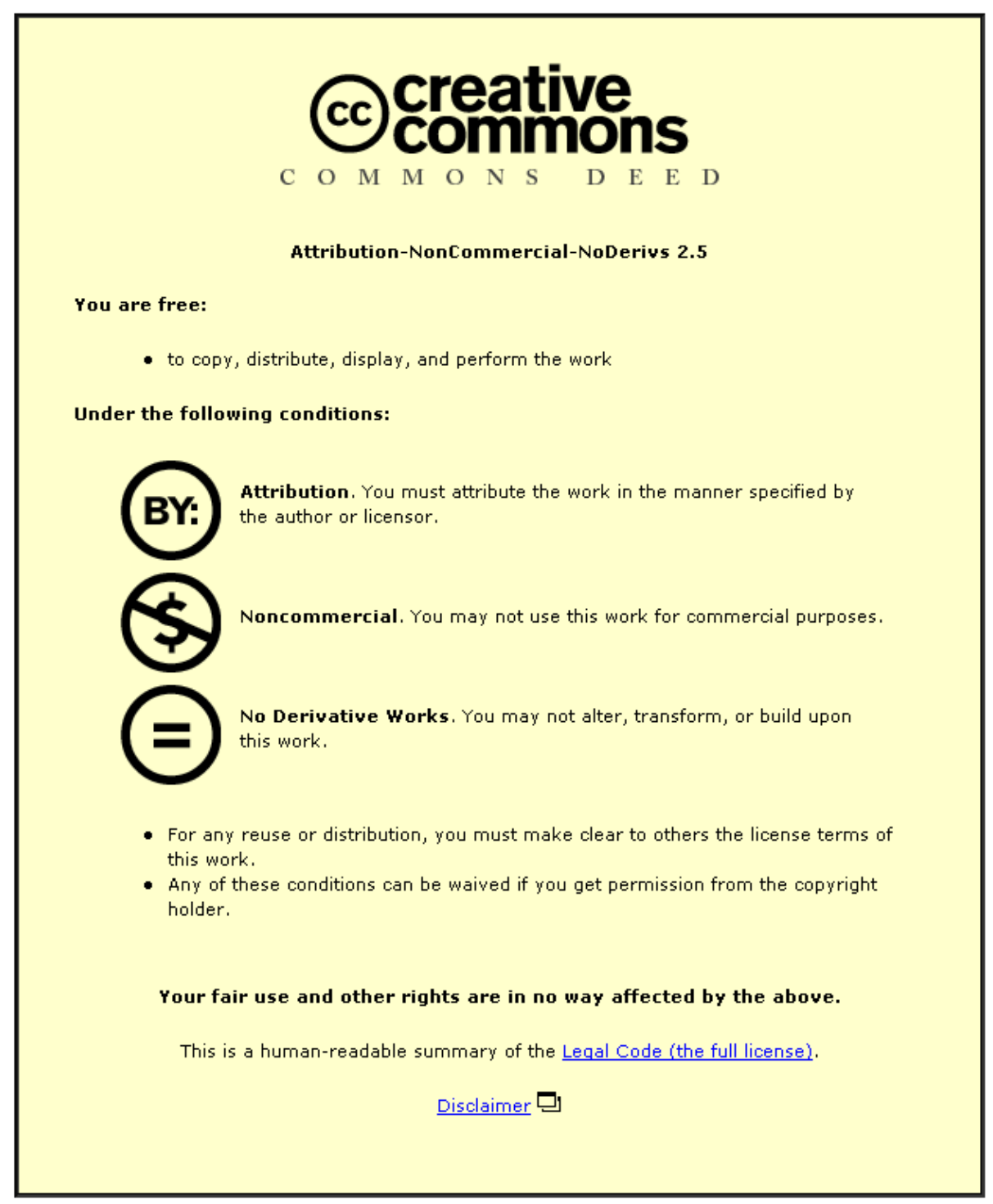

For the full text of this licence, please go to: http://creativecommons.org/licenses/by-nc-nd/2.5/ 


\title{
Experimental confirmation of the propulsion of marine vessels
}

\author{
employing guided flexural waves in attached elastic fins
}

\author{
V.V. Krylov*, G.V. Pritchard \\ Department of Aeronautical and Automotive Engineering, \\ Loughborough University, \\ Loughborough, Leicestershire LE11 3TU, UK
}

\section{Abstract}

This paper describes the results of the first experimental verification of the idea of wavelike aquatic propulsion of manned marine vessels first published by one of the present authors (V.V.K.) in 1994. The idea is based on employing the unique type of guided flexural elastic waves propagating along edges of immersed wedge-like structures attached to a body of a small ship or a submarine as keels or wings and used for the propulsion. The principle of employing such guided flexural waves as a source of aquatic propulsion is similar to that used in nature by stingrays. It is vitally important for the application of this idea to manned vessels that, in spite of vibration of the fins, the main body of the craft remains undisturbed as the energy of guided elastic waves is concentrated away from it. The main expected advantages of this new propulsion method over the existing ones, e.g. jets and propellers, are the following: it is quiet, and it is environmentally friendly and safe for people and wildlife. To 
verify the idea experimentally, the first working prototype of a small catamaran using the above-mentioned wave-like propulsion via the attached rubber keel has been build and tested. The test results have shown that the catamaran was propelled efficiently and could achieve the speed of $36 \mathrm{~cm} / \mathrm{s}$, thus demonstrating that the idea of wave-like propulsion of manned craft is viable. The reported proof of the viability of this idea may open new opportunities for marine craft propulsion, which can have far reaching implications.

Key words: Wave-like aquatic propulsion, wedge elastic waves, experimental testing.

\section{Introduction}

Emulating fish modes of swimming to man-made marine craft has been tried extensively in the past. Botman (1965) investigated the feasibility of using a mechanically excited cantilevered undulating plate to propel a model catamaran, with the direction of wave propagation being opposite to the direction of swimming. He has demonstrated experimentally that this type of propulsion is feasible, albeit not very efficient in comparison with a propeller. However, due to a number of advantages over a propeller, it may find some useful special applications. Among such advantages, Botman (1965) mentions the absence of shaft-sealing problem (which is regarded as serious for a propeller under the high hydrostatic pressures occurring at great depth). Other important advantages are low underwater acoustic noise (due to the absence of cavitation normally associated with a propeller), safe environment for swimmers, small idling drag and good thrust control. 
Paidoussis (1976) used a similar model catamaran with a submerged undulating plate. However, the propagating plate flexural waves in his experiments were excited not mechanically, but as a result of hydroelastic instability (flutter) induced by a fluid flow of sufficiently high velocity supplied from a flexible pipe and interacting with the plate. The propulsion due to undulating plate was thus added to the propulsion induced by fluid jet. Speaking of the advantages of this method of wave-like propulsion, Paidoussis (1976) also mentions the possibility of avoiding sealing of rotating propeller shafts in deep water applications. In addition, he indicates the ability of the method to propel vessels in muddy and weed-infested environments. Note that in both investigations mentioned above the authors tried to emulate the so-called 'anguilliform' fish swimming mode, which is a subcategory of the more general type of body and/or caudal fin locomotion (BCF) used most extensively for propulsion in nature (Stakiotakis et al., 1999; Paidoussis, 2004; Willy and Low, 2005).

Subsequent researchers subjected the anguilliform and other types of BCF locomotion to a great deal of investigations and engineering imitations, using undulating plates and simple oscillators as well as smart actuators (Triantafyllou and Triantafyllou, 1995; Triantafyllou et al., 2000; Yamamoto et al., 1995; Sfakiotakis et al., 1999; Wolfgang et al., 1999; Guo et al. 2003; Guglielmini et al., 2003; Schouveiler et al., 2005). One should note, however, that practical applications of anguilliform and other types of BCF propulsion are limited to unmanned autonomous underwater vehicles (AUV), which are used widely for research and surveillance operations. For manned vessels the anguilliform and BCF propulsion is unsuitable, as the main body of the vessel would be subject to intensive vibrations in reaction to the propulsion, which would make onboard conditions unsustainable. For that reason, emulating the so-called median and/or paired fin (MPF) locomotion seems to be more attractive for manned vessels, one of the subcategories of this locomotion being called 'rajiform' used by stingrays and skates (Stakiotakis et al., 1999). 
Stingrays and skates have large triangular pectoral fins that extend over the entire length of their body. Thrust is produced by large-amplitude undulations of pectoral fins propagating from the anterior to the posterior of the fish (Sfakiotakis et al., 1999). It is this specific undulatory swimming mode used by stingrays that is inspirational and the most close to the wave-like propulsion employing wedge elastic waves described in the present work. Note in this connection that a working prototype of a robot emulating undulations of pectoral fins of a cuttlefish has been recently described by Willy and Low (2005). However, in their design Willy and Low synthesised wave-like motion of each of the two pectoral fins (wings) using a complex mechanical system of connected acrylic sheets driven by 10 servomotors making one wavelength per fin due to the phase shifts between rotations of the servomotors. In contrast to the above-mentioned cuttlefish robot using phase-shifted servomotors, the method of aquatic propulsion investigated in this paper is based on the natural mode of wave motion present in wedge-like structures, which makes this method much more simple and more reliable.

The idea of wave-like aquatic propulsion of manned vessels to be described in this paper has been first published by Krylov (1994). This idea is based on employing the unique type of localised flexural elastic waves propagating along edges of wedge-like structures immersed in water (wedge elastic waves). Such wedge-like structures supporting localised elastic waves are to be attached to a body of a small ship or a submarine as keels or wings that are to be used for aquatic propulsion (Fig. 1). The above-mentioned wedge elastic waves propagating in contact with water have been first predicted and investigated theoretically (Krylov, 1994, 1998) and later confirmed in the independent experiments (Chamuel, 1996; De Billy, 1996). Obviously, the principle of using localised elastic waves as a source of aquatic propulsion is similar to that used in nature by stingrays, which utilise wave-like motions of their large horizontal pectoral fins (wings) for moving forward. It is vitally important for the application of wedge elastic waves for propulsion of manned marine vessels that, in spite of vibration of 
the fins, the main body of the craft remains undisturbed because the energy of localised elastic waves is concentrated near the wings’ tips (Krylov, 1994).

Thus, the main advantage of the new wave-like propulsion method of marine craft over the existing methods using undulating plates, e.g. the ones described by Botman (1965) and Paidoussis (1976), is that the main body of the craft is isolated from fin vibrations. This permits this method to be used for propulsion of manned vessels.

Other advantages of the method should be considered in comparison with traditional methods of propulsion, such as jets and propellers. They are largely the same as those mentioned by Botman (1965) and Paidoussis (1976). Namely, the absence of propeller shaftsealing problem at large depth, low underwater acoustic noise (due to the absence of cavitation normally associated with a propeller), safe environment for swimmers, small idling drag, good thrust control, and the ability to propel vessels in muddy and weed-infested environments.

The main envisaged applications of the proposed type of wave-like propulsion are small and medium manned research submarines and autonomous underwater vehicles (AUV). Another possible application is sailing. As it is well known, sailing boats are dependent on the wind for propulsion and are therefore stranded in very calm conditions. This problem is usually overcome with outboard motors for use in times of low wind. The replacement of the outboard motor by a flexible keel harnessing wave-like propulsion, in place of the centre/dagger board, would have significant advantages. In particular, the propulsive keel (fin) would not interfere with the hydrodynamic characteristics of the hull.

In the present paper we report the results of the first experimental validation of the abovementioned idea of wave-like aquatic propulsion using guided elastic waves in attached elastic fins which is suitable for manned marine vessels. To verify the idea experimentally, we have built and tested the working prototype of a small catamaran employing the above-mentioned 
wave-like propulsion via the attached rubber keel. The tests have been carried out in two phases, in a water tank and then in open water. The test results have shown that the catamaran was propelled efficiently and could achieve the speed of around one its body length per second (36 cm/s), thus demonstrating that the idea of wave-like propulsion of manned craft is viable. The reported proof of the viability of this idea may open new opportunities for marine craft propulsion, which can have far reaching implications.

\section{Guided elastic waves in contact with water}

The wave-like aquatic propulsion suitable for manned vessels, which is the main objective of this paper, is based on employing the unique type of guided flexural waves propagating along tips of elastic wedges submerged in water (Krylov, 1994, 1998). The energy of such waves, that are often called water-loaded wedge elastic waves, is propagating

along the wedge edges but is confined (localised) in lateral direction (see Fig. 1). As was mentioned above, the confinement of the wave energy in lateral direction is paramount for use of these waves for propulsion of manned vessels, as the main body of the vessel is kept isolated from waves propagating along the fins.

Because of their complex nature, wedge waves generally can be described only numerically even for the simplest case of wedges in vacuum which was first considered back in the 1970's (see e.g. the book of Biryukov et al. (1995) and references there for more detail). This is even more so for wedges in contact with water. However, for an important case of slender wedges the situation can be simplified in both cases by using the geometrical acoustics approximation (Biryukov et al., 1995; Krylov, 1989, 1990a, 1990b). Using this approximation, one can solve the equations for bending vibrations of slender wedges of 
different shapes. As a result, one can obtain relatively simple and physically explicit solutions for localised waves propagating in wedges in contact with vacuum (Krylov, 1989, 1990a, 1990b) as well as in wedges immersed in water (Krylov, 1994, 1998; Krylov and Shuvalov, 2000; Shuvalov and Krylov, 2000). Other known approaches to analysing wedge elastic waves in contact with water include Wiener-Hopf techniques (Shanin and Krylov, 2000) and finite element calculations (Hladky-Hennion et al., 1997).

For the purpose of aquatic propulsion of manned vessels, one can use wedge waves propagating in wedges of any shapes. All of them provide localisation of the wave energy in the lateral direction. The most suitable, however, appear to be quadratic wedges, which local thickness is described by the function $h(x)=\varepsilon x^{2}$, where $x$ is the distance from the edge and $\varepsilon$ is a constant. In such wedges, all modes of guided flexural waves are dispersive, i.e. their phase velocities depend on frequency. In the case of using these waves for aquatic propulsion, this would allow an operator of a vessel to change wedge wave velocity by changing frequency, which may be convenient for efficient start and acceleration of the vessel from rest. In this connection, we remind the reader that, as follows from the theory and experimental observations of fish swimming, the wave velocity of undulating body at stationary conditions should be slightly higher than the velocity of swimming (Lighthill, 1960, 1970, 1971; Cheng et al., 1994; Muller et al., 2002). Another advantage of using quadratic wedges is that they utilise a larger proportion of their surfaces for localised wave propagation in comparison with linear wedges, which again is beneficial for aquatic propulsion.

Although waves in quadratic wedges seem to be the most appropriate for aquatic propulsion of manned vessels, it was rather difficult to use them in the present preliminary experimental investigation due to difficulties in manufacturing of experimental quadratic wedges. To avoid this problem on this initial stage it was decided to use the earlier established 
similarity between guided wave propagation in quadratic wedges and in the systems comprising thin ridges embedded into an elastic half-space (Krylov, 1990b). The latter systems in turn are similar to infinite elastic strips with one edge being free and with another one being clamped. Therefore, for experimental purposes of this paper, the 'clamped-free' rubber plates were used instead of quadratic rubber wedges. One should keep in mind however that, in contrast to quadratic wedges, such plates do transmit vibrations to the main body of a vessel. Therefore, they can not be recommended for applications in real-life manned vessels.

One should keep in mind that all of the above-mentioned guided elastic waves in contact with water are waves propagating in the subsonic regime of wave propagation (in comparison with the speed of sound in water). As it is well known, such waves do not generate sound in the surrounding water. Adding to this the absence of cavitation normally associated with propellers, one could expect that the proposed wave-like propulsion is much quieter than a propeller.

Figure 2 shows the theoretically calculated frequency-dependent phase velocities (dispersion curves) of the lowest-order flexural guided mode in an immersed 'clamped-free' rubber strip. Calculations have been carried out using the geometrical acoustics approach (Krylov, 1994, 1998) for the two values of strip thickness: $1 \mathrm{~mm}$ and $3 \mathrm{~mm}$. The width of the strip was $150 \mathrm{~mm}$ in both cases. For comparison, the corresponding phase velocities of flexural waves in infinite immersed plates are shown as well. One can see that for the strip of $1 \mathrm{~mm}$ thickness the dispersion curves for a finite strip and for an infinite plate are almost indistinguishable, whereas the phase velocity for a $3 \mathrm{~mm}$ strip is slightly higher and has a minimum at frequency of around $0.3 \mathrm{~Hz}$. 


\section{Test rig design}

According to the previous section, to generate wave like propulsion of a small model of a vessel that would imitate the real manned marine vessel it was sufficient to use elastic rubber plates clamped on one side, instead of using quadratic rubber wedges. The plate was to be excited at a single point, with the resulting flexural waves propagating in the direction opposite to the direction of swimming. Although the main envisaged applications for the proposed wave-like propulsion is for use on ocean research submarines, at this early stage of the investigation a simpler surface boat type rig has been designed and built.

The basic rig comprised the vertical propulsive rubber plate (fin) emulating the 'balistiform' mode of fish propulsion (a subcategory of the MPF locomotion). The plate was mounted on the two supporting pylons (30 $\mathrm{cm}$ each) positioned at 90 degrees to the plate (see Fig. 3). The pylons could then be either clamped above a water tank or their ends could be attached to a pair of pontoons to form a catamaran. The propulsive rubber plate was clamped along one edge and was free on the other three sides. The plate was excited at the tip by a mechanical pivoted arm that was attached to the leading edge of the propelling plate and driven by the electric motor. The motor was connected to the arm via a gearbox and a disk with a set of 12 drilled holes at different distances from the centre of rotation, allowing a change in amplitude of the plate excitation by changing the pivot position. These holes were designated by the symbols D1 - D12, each of them being associated with the certain amplitude of displacement of the plate tip.

The experimental testing took place in two stages. The first stage was carried out in a water tank, where the propulsive rig did not move. This testing in an enclosed and controlled environment allowed for easier and more accurate observations of the waves in a propulsive 
fin and of the wave-generated water flows associated with the propulsion. The second stage took place in open water, with the moving rig mounted on a catamaran.

Assuming that frequencies of wave excitation were in the range $2-10 \mathrm{~Hz}$, the thickness of the basic rubber plate was chosen as $1 \mathrm{~mm}$. The width (span) and the length (chord) of the propulsive rubber plate were chosen as $150 \mathrm{~mm}$ and $250 \mathrm{~mm}$ respectively. To optimise the thickness of the propulsive fin, in addition to the basic plate of $1 \mathrm{~mm}$ thickness (see previous section), two thicker pieces of rubber of the same dimensions were tested. The thickest was of the order of $3 \mathrm{~mm}$ thick, and the thinner was approximately $2 \mathrm{~mm}$ thick. The above-mentioned three rubber samples will be referred to as thin (1mm), medium (2mm) and thick (3mm). As expected, for operating frequencies within the range from 2.5 to $4.5 \mathrm{~Hz}$, a complete flexural wavelength was not observed along the chord of the thick rubber plate, and the fin simply acted as a side-to-side paddle. On the other hand, the most flexible thin sample (1 mm) allowed for a number of wavelengths to be observed along the chord of the fin.

A 12-24 V dc motor with a gearbox was chosen to power the craft. In particular, using a 64:1 gear ratio gave a suitable maximum rotational speed of roughly $300 \mathrm{rpm}($ or $5 \mathrm{~Hz}$ ) at the maximum voltage of $24 \mathrm{~V}$.

A clear plastic water container was used for the in-tank testing. The twin pylons supporting the fin and motor were sized to fit by bolts exactly across the top of the container. Water was added to the container to submerge the fin span.

The open-water stage of testing incorporated the same basic test rig as the water-tank testing, with the addition of buoyancy aids (two Styrofoam pontoons) to the sides of the rig, thus assembling a catamaran craft. The design of the complete open-water test rig (a catamaran) is demonstrated in Fig. 4, and its real view is shown in Fig.5. As was mentioned above, the basic rig, with the exception of pontoons, was essentially the same for both watertank and open-water tests. 
The pontoons were designed to maintain the fully immersed water depth of the fin. With the motor mass offset from centre in both the longitudinal and lateral directions, the righthand pontoon needed to be longer than the left (50 cm and $35 \mathrm{~cm}$ respectively), with both pontoons extending forward of the main deck. This configuration ensured a level deck of the model craft.

\section{Experimental results}

The initial testing took place in a water tank, with the propulsive fin placed vertically in the centre of the container. The wave-generated water flow speeds were measured by timing the movement of small pieces of cork over a set distance. The aim of this testing was to observe the behaviour of the fin over a range of amplitudes and frequencies. The frequency of wave excitation was defined by a number of motor revolutions per second, which in turn depended on the loading and on the input electric power. Increasing the voltage increased the power, and therefore increased the motor speed of rotation and frequency of the fin vibrations.

Results were obtained for different values of fin vibration amplitudes at various voltage settings influencing the frequency of vibration. The speed of the water flow generated by flexural waves propagating along the propulsive fin was measured for different values of the input electric power and frequency. Results were recorded for 4 values of fin vibration amplitudes at 11 voltage settings, from $15 \mathrm{~V}$ to $25 \mathrm{~V}$ at 1 -volt intervals, changing the frequency of fin vibration. It has been found that within the used frequency range (2.4-4.5 Hz) water flow speeds generally increased with the increase of input power (or frequency) from $0.7 \mathrm{~cm} / \mathrm{s}$ to about $11 \mathrm{~cm} / \mathrm{s}$. In particular, it has been concluded by extrapolation of these results that flow was not to be generated for values of input power below about $0.8 \mathrm{~W}$, which corresponds 
to the value of the critical driving frequency of about $2.0 \mathrm{~Hz}$. Note that the observed flow speeds in the water tank are comparable with the calculated phase velocities of guided flexural waves propagating along the propulsive rubber strips (see Fig. 2).

Naturally, the best operating setting for sustained running of the model vessel in open water would be the one corresponding to the maximum efficiency of propulsion. Using the measured values of the input electric power $P_{\text {in }}=I \cdot V$ and of the flow speed $v$ in a water tank, it was possible to estimate relative values of the propulsive efficiency of the fin at the various test conditions. Assuming that wave-generated thrust at stationary condition is equal to the combined drag produced by the fin, container and all other surfaces and is proportional to the square of the generated flow speed (Batchelor, 1994), the efficiency $\eta$ can be calculated using the following simple formula:

$$
\eta=\frac{P_{\text {out }}}{P_{\text {in }}}=\text { const } \cdot \frac{v^{3}}{I \cdot V}
$$

where $P_{\text {out }}$ is the output power associated with the fin-generated water flow and calculated as the product of the associated thrust produced by the fin and the flow velocity. Note that there is still an unknown constant in the equation (1) depending on the parameters of the rig. Therefore, equation (1) can be only used for relative comparison of the efficiencies for the same experimental rig, e.g. by normalising them with respect to the maximum efficiency achieved. The relative value of efficiency normalised in this way can be referred to as the relative efficiency.

The relative efficiency as function of frequency (or input power) has been calculated according to (1) using the measured values of $v$ and $I, V$. In particular, it has been found that the relative efficiency depends on frequency and on the wave amplitude, changing in values from almost zero to $100 \%$. The maximum relative efficiency (100\%) occurred at 3.8 Hz, with the amplitude setting D6 (at the outermost hole 6 on the disc; this corresponds to the 
plate tip displacement amplitude $\mathrm{W}_{0}=2.8 \mathrm{~cm}$ ). The lowest efficiency (almost zero) occurred at $3.3 \mathrm{~Hz}$ for the amplitude setting $\mathrm{D} 2$ (or $\mathrm{W}_{0}=1.8 \mathrm{~cm}$ ). The frequencies at which the highest and lowest efficiencies have occurred correspond to input voltages of $23 \mathrm{~V}$ and $18 \mathrm{~V}$ respectively. At the above-mentioned amplitude setting D6 and voltage $23 \mathrm{~V}$ corresponding to the maximum efficiency the system was consuming the input electric power $\mathrm{P}_{\text {in }}=5 \mathrm{~W}$. Using this relatively high power at open-water testing would have drained a series of small electric batteries on board the craft very quickly. The alternative was to use large lead acid batteries for the open-water testing. These were very heavy and required to be off the craft, with a wire feed.

The open-water testing was carried out in the experimental pool for the assembled small model catamaran with the guided wave propulsion. The view of the moving model catamaran is shown in Fig. 6 (note that the propulsive rubber plate is not visible in Fig. 6 since it is below water level). Although the open-water testing was designed mainly to prove the idea and not to gain accurate results, the craft nonetheless was tested in several configurations. Two sets of tests were performed. The first was a timed run over a distance of $3 \mathrm{~m}$, the craft starting form rest. This was designed to measure averaged speeds of the craft over this distance. The second set of tests was again a timed run over a distance of $2 \mathrm{~m}$, but with an initial speed achieved after passing the added 'acceleration' distance of 2m, an ample space to reach a stationary speed. This set of tests was designed to measure stationary speeds. The frequency in all tests was maintained at the value corresponding to the maximum relative efficiency determined from the in-tank testing (around $3.8 \mathrm{~Hz}$ ), whereas the wave amplitudes were variable.

In addition to the three black rubber plates tested in a water tank, a white rubber plate was added for open-water testing. This white rubber was of the same thickness as the thin black rubber, but was less stiff. As in the water-tank tests, a single rubber plate was used (1 sheet) 
with dimensions 250x150mm, or one could use a rubber plate of the same size supported by two smaller side plates (3 sheets) with dimensions 70x250 mm.

When powered from the external batteries via a flexible cable, the catamaran demonstrated fast acceleration from rest to stationary speeds that were high enough in terms of its lengths per second (see Fig. 6). The results of the two sets of tests for different configurations are presented in Tables 1 and 2. They have been arranged in time order, the fastest at the top, with an averaged time (and the resulting averaged speed) obtained over the three runs. The amplitude settings were D4 $\left(\mathrm{W}_{0}=2.2 \mathrm{~cm}\right), \mathrm{D} 6\left(\mathrm{~W}_{0}=2.8 \mathrm{~cm}\right)$ and D8 $\left(\mathrm{W}_{0}=\right.$ $3.2 \mathrm{~cm})$. The obtained results demonstrate that the achieved stationary speeds are high enough in terms of the craft body length, which means that the proposed wave-like propulsion is viable.

Note that the parallel-sided rubber plate performed more efficiently when flanked by additional smaller rubber sheets. A possible explanation for this could be that a sided plate, being stiffer, has a higher wave velocity, thus resulting in a higher stationary swim speed.

The comparison of the speeds of normal and heavier craft (with additional weight) has shown, as expected, that the normal craft performed better than the heavier one as it sat further out of the water and therefore produced less drag.

As expected, the results on relative propulsion efficiency obtained from the in-tank testing agree with those observed in the open-water test (based on the comparison of the catamaran speeds at the same input power). For example, at the open-water test condition of 22 volts, the most efficient amplitude setting was D6, then D8, and then D4. This was fully replicated in the in-tank testing.

Absolute measurements of the propulsive efficiency and comparison of the efficiency of the proposed type of wave propulsion with the efficiency of a propeller were beyond the scope of this paper concerned primarily with the feasibility studies. To make such 
measurements and the comparison meaningful one would require to optimise the mechanical design of the model vessel. In the current design, which employs the motor disk and mechanical arms for flexural wave generation, a substantial amount of energy is being lost due to friction at the mechanical arm contact points. This reduces substantially the energy efficiency of the system.

It makes sense, however, to evaluate the hydrodynamic efficiency of the proposed wave propulsion indirectly, using the non-dimensional Strouhal number $S t=f W_{0} / U$, where $f$ is the undulating wave frequency, $W_{0}$ is the wave amplitude, and $U$ is the stationary speed achieved by the vessel. It is known that the Strouhal number can be used to characterise the propulsive efficiency of fish regardless of the energy consumption used to achieve the actual values of $f, W_{0}$, and $U$. (see e.g. Taylor et al., 2003). It is also known that propulsive efficiency is the highest for St within the interval $0.2<\mathrm{St}<0.4$. In particular, this is true for such efficient swimmers as dolphins, sharks and bony fish, which all swim at $0.2<\mathrm{St}<0.4$.

Note in this connection that the value of St for the proposed wave-like propulsion in the regime corresponding to its maximum relative efficiency $\left(\mathrm{f}=3.8 \mathrm{~Hz}, \mathrm{~W}_{0}=2.8 \mathrm{~cm}, \mathrm{U}=35.8\right.$ $\mathrm{cm} / \mathrm{s}$ ) can be calculated as $\mathrm{St}=3.82 .8 / 35.8=0.297$. The remarkable fact is that this value of St is also in the 'high efficiency range' $0.2<\mathrm{St}<0.4$, which apparently implies that the proposed wave like propulsion is potentially as efficient as the propulsion of dolphins and sharks. In the future design, an improved version of the model vessel could be developed, e.g. using electro-active bending polymers (EAP) to directly generate flexural waves at a leading edge in a plate or wedge, instead of the mechanical arms. This would reduce or even eliminate any moving parts from the exciting system. It is expected that energy efficiency of such an improved vessel would be essentially higher than that of the current model catamaran, possibly approaching the efficiency of dolphins and sharks. 


\section{Conclusions}

The most important conclusion resulting from this work is that guided wedge or plate elastic waves can indeed be used to propel a model marine craft. Moreover, due to the wedge wave energy concentration away from the main body of the craft, such waves are suitable for propelling real manned marine vessels.

A wave propulsion rig has been implemented that produces thrust both in the controlled water-tank test environment and in the open water. It has been also shown that the propulsion is operable over a continuum of speeds, including acceleration from rest, and that its efficiency can be maximised by changing the amplitude and frequency of the input force.

The speeds achieved by the small model catamaran in the open water testing are comparable with the wave speeds in the propulsive plate at the operating frequencies. This is in agreement with the well-known results following from the studies of fish swimming indicating that speed of swimming at stationary conditions should be slightly less than the velocity of wave-like motion of the fish body.

Although the current experimental rig used a rather complex mechanical construction to achieve the guided flexural wave excitation, it is expected that further investigations could lead to the development of simpler and more efficient marine craft. In particular, this could include the use of electro-active bending polymers (EAP) to generate flexural waves at a leading edge in a plate or wedge, instead of the mechanical exciter used in this work. This would reduce or even eliminate any moving parts from a wave propulsion rig, giving it another advantage over a conventional propeller.

Further work is required to investigate the efficiency of wave propulsion in comparison with its main rival, a propeller. However, even if the efficiency of wave-like propulsion 
cannot be developed to surpass that of the propeller, there is still an unexplored niche for it. Namely, wave-like propulsion may have no rivals in cases where quiet and safe operation is paramount. 


\section{References}

Batchelor, G.K., 1994. An introduction to fluid dynamics. Cambridge University Press, Cambridge.

Biryukov, S.V., Gulyaev, Yu.V., Krylov, V.V., Plessky, V.P., 1995. Surface Acoustic Waves in Inhomogeneous Media. Springer-Verlag, Berlin, Heidelberg.

Botman, M., 1965. Propulsion by undulating plates. Journal of Aircraft 2, 456-462.

Chamuel, J.R., 1996. Flexural edge waves along free and immersed elastic waveguides.

Review of Progress in Quantitative Nondestructive Evaluation, Vol.16, ed. D.O.

Thompson and D.E. Chimenti (Proc. 16th Symp. Quant. Nondestruct. Eval., July 28August 2, 1996, Brunswick, Maine), Plenum Press, New York, 129-136.

Cheng, J.Y., Blikhan, R., 1994. Note on the calculation of propeller efficiency using elongated body theory. Journal of Experimental Biology 192, 169-177.

De Billy, M, 1996. On influence of loading on the velocity of guided acoustic waves propagating in linear elastic wedges. Journal of the Acoustical Society of America 100, 659-662.

Guglielmini, L., Blondeaux, P., Vittori, G., 2003. A simple model of propulsive oscillating foils. Ocean Engineering 31, 883-899.

Guo, S., Fukuda, T., Asaka, K., 2003. A new type of fish-like underwater microrobot. IEEE/ASME Transactions on Mechatronics 8, 136-141.

Hladky-Hennion, A.-C., Langlet, P., De Billy, M., 1997. Finite element analysis of the propagation of acoustic waves along waveguides immersed in water. Journal of Sound and Vibration 200, 519-530. 
Krylov, V.V., 1989. Conditions for validity of the geometrical-acoustics approximation in application to waves in an acute-angle solid wedge. Soviet Physics - Acoustics 35, 176180.

Krylov, V.V., 1990a. Geometrical-acoustics approach to the description of localized vibrational modes of an elastic solid wedge. Soviet Physics - Technical Physics 35, 137140.

Krylov, V.V., 1990b. Localized acoustic modes of a quadratically-shaped solid wedge. Moscow University Physics Bulletin 45(6), 65-69.

Krylov, V.V., 1994. Propagation of wedge acoustic waves along wedges embedded in water. Proc. IEEE Ultrasonics Symposium, Cannes, France, 793-796.

Krylov, V.V., 1998. On the velocities of localized vibration modes in immersed solid wedges. Journal of the Acoustical Society of America 103, 767-770.

Krylov, V.V., Shuvalov, A.L., 2000. Propagation of localised flexural vibrations along plate edges described by a power law, Proceedings of the Institute of Acoustics 22(2), 263270.

Lighthill, M.J., 1960. Note on the swimming of slender fish. Journal of Fluid Mechanics 9, 305-317.

Lighthill, M.J., 1970. Aquatic animal propulsion of high hydrodynamic efficiency. Journal of Fluid Mechanics 44, 265-301.

Lighthill, M.J., 1971. Large amplitude elongated-body theory of fish locomotion. Proceedings of the Royal Society (London) B 179, 125-138.

Muller, U.K., Stamhuis, E.J., Videler, J.J., 2002. Integrative and Comparative Biology 42(5), 981-987.

Paidoussis, M.P., 1976. Hydroelastic ichthyoid propulsion. Journal of Hydronautics 10, 3032. 
Paidoussis, M.P., 2004. Fluid-Structure Interactions, Vol. 2. Elsevier, Section 8.10.

Sfakiotakis, M., Lane, D.M., Davies, J.B.C., 1999. Review of fish swimming modes for aquatic locomotion. IEEE Journal of Oceanic Engineering 24, 237-252.

Shanin, A.V., Krylov, V.V., 2000. An approximate theory for waves in a slender elastic wedge immersed in liquid. Proceedings of the Royal Society (London) A 456, 21792196.

Schouveiler, L., Hover, F.S., Triantafyllou, M.S., 2005. Performance of flapping foil propulsion. Journal of Fluids and Structures 20, 949-959.

Shuvalov, A.L., Krylov, V.V., 2000. Geometrical-acoustics consideration of the flexural modes in immersed anisotropic wedges. Journal of Sound and Vibration 237, 427-434.

Taylor, G.K., Nudds, R.L., Thomas, A.L.R., 2003. Flying and swimming animals cruise at a Strouhal number tuned for high power efficiency. Nature 425, 707-711.

Triantafyllou, M.S., Triantafyllou, G.S., 1995. An efficient swimming machine. Scientific American 272, 64-70.

Triantafyllou, M.S., Triantafyllou, G.S., Yue, D.K.P., 2000. Hydrodynamics of fishlike swimming. Annual Review of Fluid Mechanics 32, 33-53.

Willy, A., Low, K.H., 2005. Undulating fins of a cuttlefish robot. http://releeps.tripod.com/cgi-bin/

Wolfgang, M.J., Yue, D.K.P., Triantafyllou, M.S., 1999. Visualisation of complex near-body transport processes in flexible-body propulsion. Journal of Flow Visualisation 2, 143151.

Yamamoto, I., Terada, Y., Nagamatu, T., Imaizumi, Y., 1995. Propulsion system with flexible/rigid oscillating fin. IEEE Journal of Oceanic Engineering 20, 23-30. 


\section{List of Figures}

Fig. 1. Artist's impression of the proposed use of guided elastic waves for propulsion of a small submarine (after Krylov (1994)); the guided flexural waves propagate along the tips of the horizontal fins (wings), their energy being concentrated away from the main body.

Fig. 2. Calculated phase velocities of the lowest order flexural mode in two 'clamped-free' water-loaded rubber strips as functions of frequency; the strips are of the same width $H=150 \mathrm{~mm}$, but have different values of thickness: $h=1 \mathrm{~mm}$ (solid curve) and $h=$ $3 \mathrm{~mm}$ (dashed curve); for comparison, the velocities of flexural waves in infinite plates are also shown for $h=1 \mathrm{~mm}$ (dotted curve) and $h=3 \mathrm{~mm}$ (dash-dotted curve).

Fig. 3. Design of the experimental propulsive plate which is bolt-clamped at the top edge (AA) and excited by a mechanical arm via a clip (BB) at the bottom edge: (a) - top elevation view; (b) - side elevation view; (c) - rear elevation view; guided elastic wave propagation is shown schematically by arrow.

Fig. 4. Design of the catamaran with the wave propulsive plate: dimensions of the pontoons (shaded parts) are given in mm; the propulsive plate is shown detached from the mechanical arm. 
Fig. 5. View of the assembled catamaran with wedge wave propulsive system before its testing in open water (the mechanical arm is detached from the propulsive rubber plate).

Fig. 6. View of the moving catamaran with guided elastic wave propulsive system during its testing in open water. 


\section{List of Tables}

Table 1. Open-water testing from rest

Table 2. Open-water testing with initial craft speed 


\section{Tables}

Table 1. Open-water testing from rest

\begin{tabular}{|c|c|c|c|c|c|c|}
\hline $\begin{array}{c}\text { Number of } \\
\text { Fin Sheets }\end{array}$ & $\begin{array}{c}\text { Central } \\
\text { Rubber } \\
\text { Type }\end{array}$ & $\begin{array}{c}\text { Voltage } \\
\mathbf{( V )}\end{array}$ & $\begin{array}{c}\text { Amplitude } \\
\text { setting }\end{array}$ & $\begin{array}{c}\text { Weight } \\
\text { conditions }\end{array}$ & $\begin{array}{c}\text { Time } \\
(\mathbf{s})\end{array}$ & $\begin{array}{c}\text { Speed } \\
\mathbf{( c m} / \mathbf{s})\end{array}$ \\
\hline 3 & White & 22.1 & D6 & Normal & 8.39 & 35.8 \\
\hline 3 & Thin & 22.4 & D6 & Normal & 8.74 & 34.3 \\
\hline 3 & Thin & 22.2 & D8 & Normal & 9.32 & 32.12 \\
\hline 1 & Thin & 22.1 & D8 & Normal & 9.68 & 31.0 \\
\hline 3 & Thin & 22.0 & D8 & Heavy & 10.52 & 28.5 \\
\hline 3 & Thin & 22.3 & D8 & Heavy & 10.54 & 28.5 \\
\hline 3 & Thin & 22.2 & D4 & Normal & 10.77 & 27.9 \\
\hline
\end{tabular}


Table 2. Open-water testing with initial craft speed

\begin{tabular}{|c|c|c|c|c|c|c|}
\hline $\begin{array}{c}\text { Number of } \\
\text { Fin Sheets }\end{array}$ & $\begin{array}{c}\text { Central } \\
\text { Rubber } \\
\text { Type }\end{array}$ & $\begin{array}{c}\text { Voltage } \\
\mathbf{( V )}\end{array}$ & $\begin{array}{c}\text { Amplitude } \\
\text { setting }\end{array}$ & $\begin{array}{c}\text { Weight } \\
\text { conditions }\end{array}$ & $\begin{array}{c}\text { Time } \\
\text { (s) }\end{array}$ & $\begin{array}{c}\text { Speed } \\
\text { (cm/s) }\end{array}$ \\
\hline 3 & White & 21.9 & D6 & Normal & 5.73 & 34.9 \\
\hline 3 & White & 22.0 & D6 & Heavy & 7.28 & 27.5 \\
\hline 3 & White & 22.0 & D6 & Heavy & 7.39 & 27.1 \\
\hline
\end{tabular}




\section{Figures}

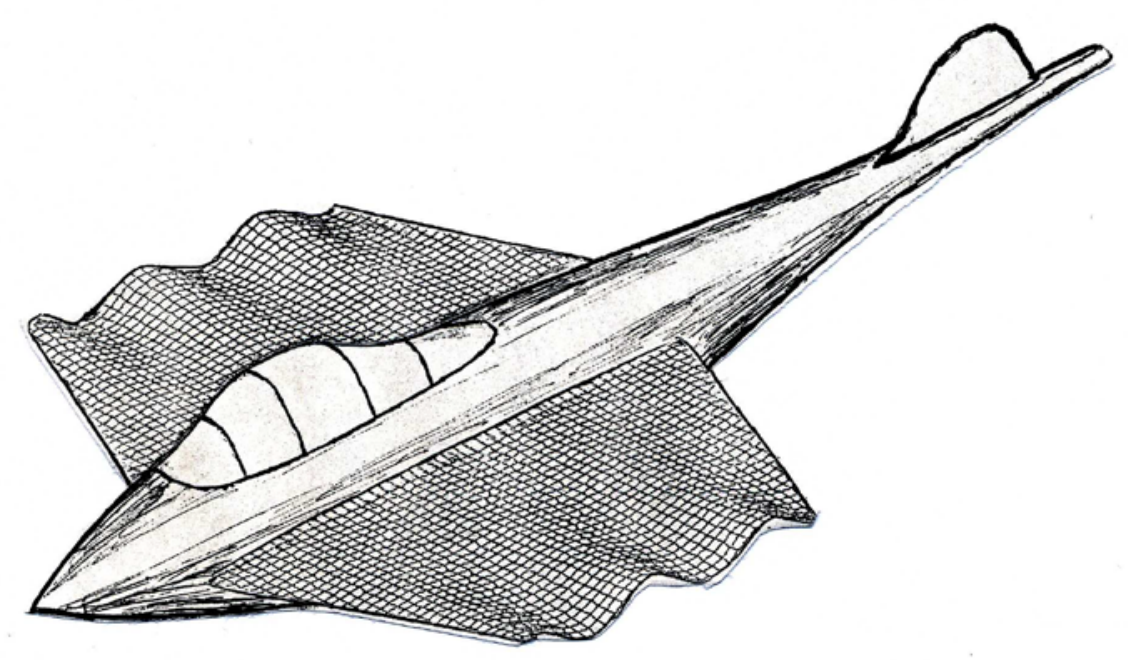

Fig. 1. Artist's impression of the proposed use of guided elastic waves for propulsion of a small submarine (after Krylov (1994)); the guided flexural waves propagate along the tips of the horizontal fins (wings), their energy being concentrated away from the main body. 


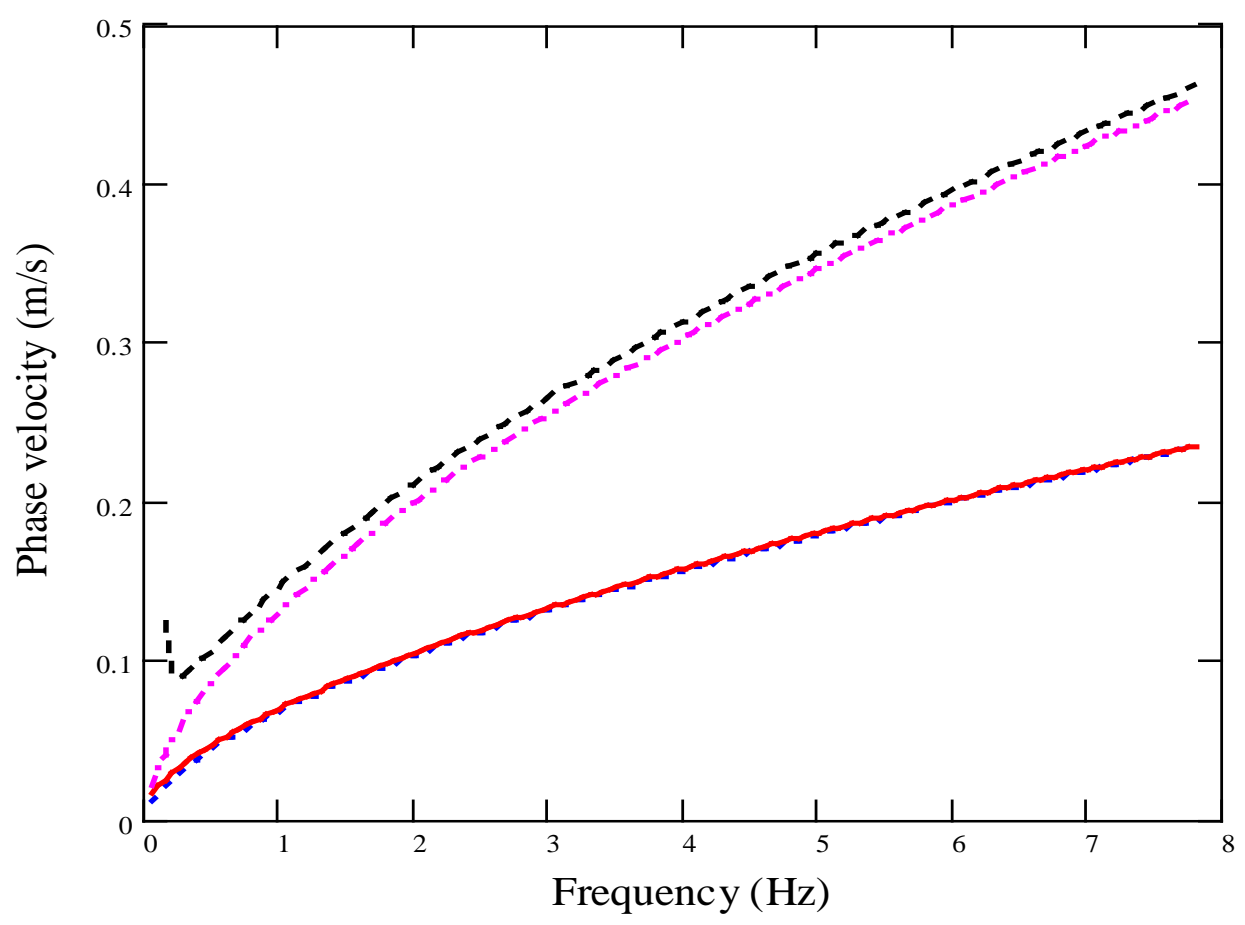

Fig. 2. Calculated phase velocities of the lowest order flexural mode in two 'clamped-free' water-loaded rubber strips as functions of frequency; the strips are of the same width $H=150 \mathrm{~mm}$, but have different values of thickness: $h=1 \mathrm{~mm}$ (solid curve) and $h$ = $3 \mathrm{~mm}$ (dashed curve); for comparison, the velocities for infinite plates are shown for $h=1 \mathrm{~mm}$ (dotted curve) and $h=3 \mathrm{~mm}$ (dash-dotted curve). 
(a) Two 30-mm pylons

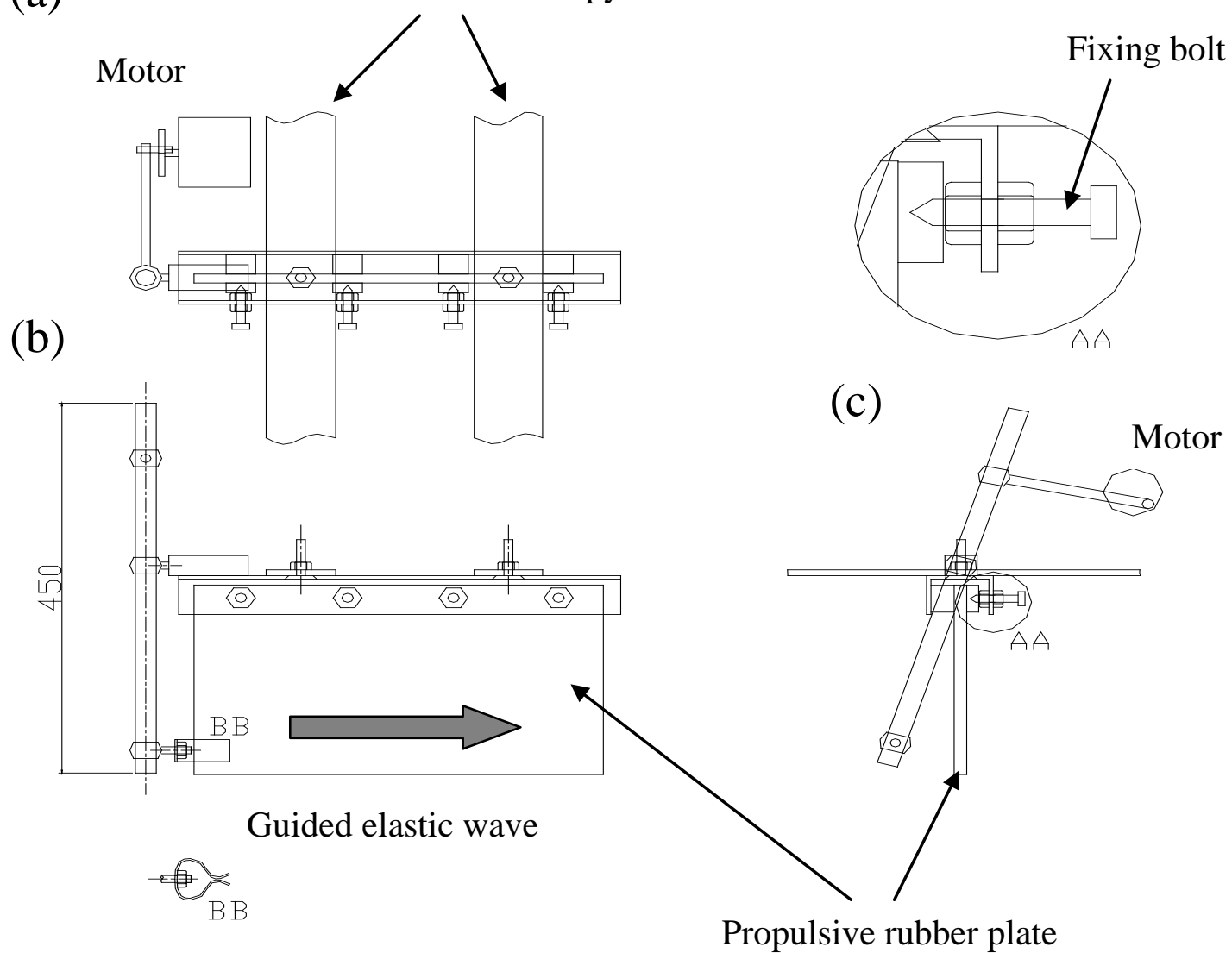

Fig. 3. Design of the experimental propulsive plate which is bolt-clamped at the top edge (AA) and excited by a mechanical arm via a clip (BB) at the bottom edge: (a) - top elevation view; (b) - side elevation view; (c) - rear elevation view; guided elastic wave propagation is shown schematically by arrow. 


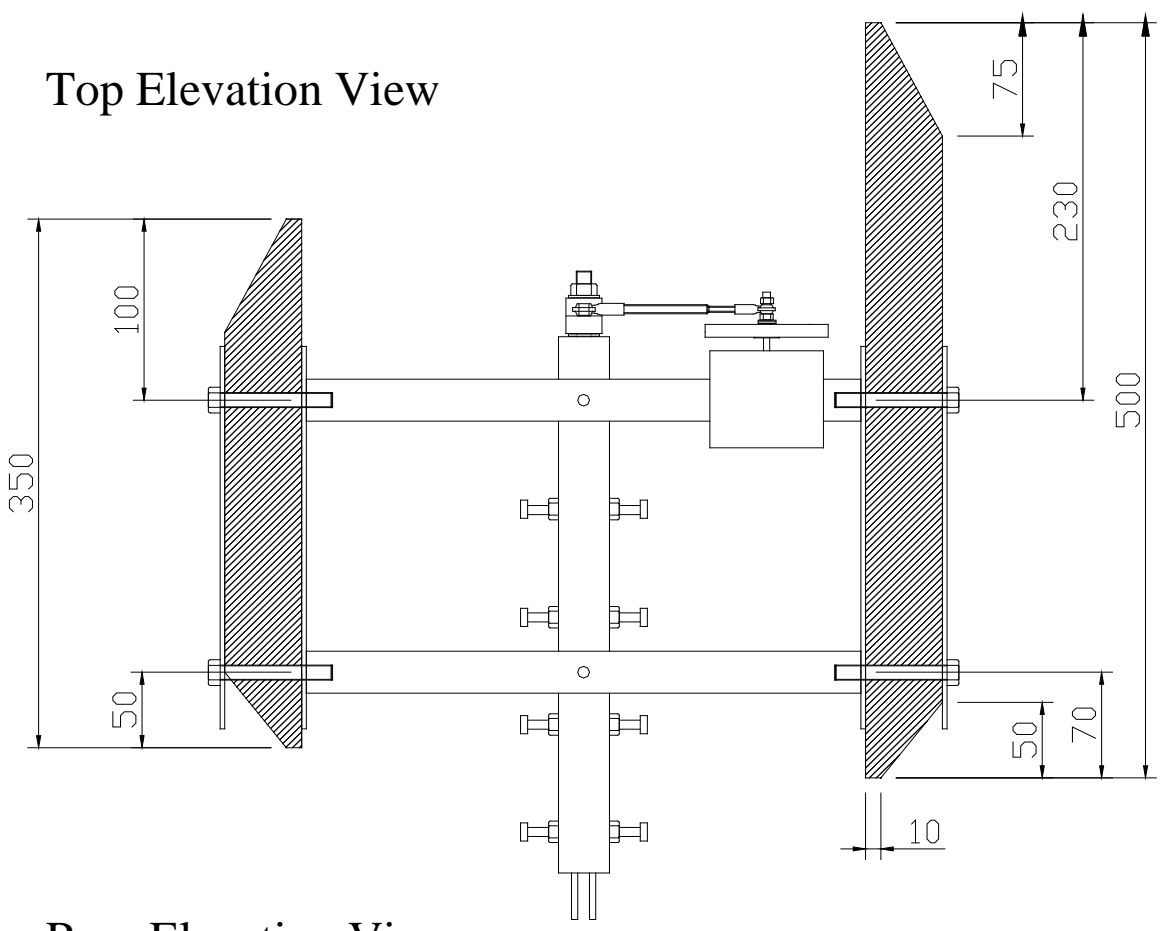

Rear Elevation View

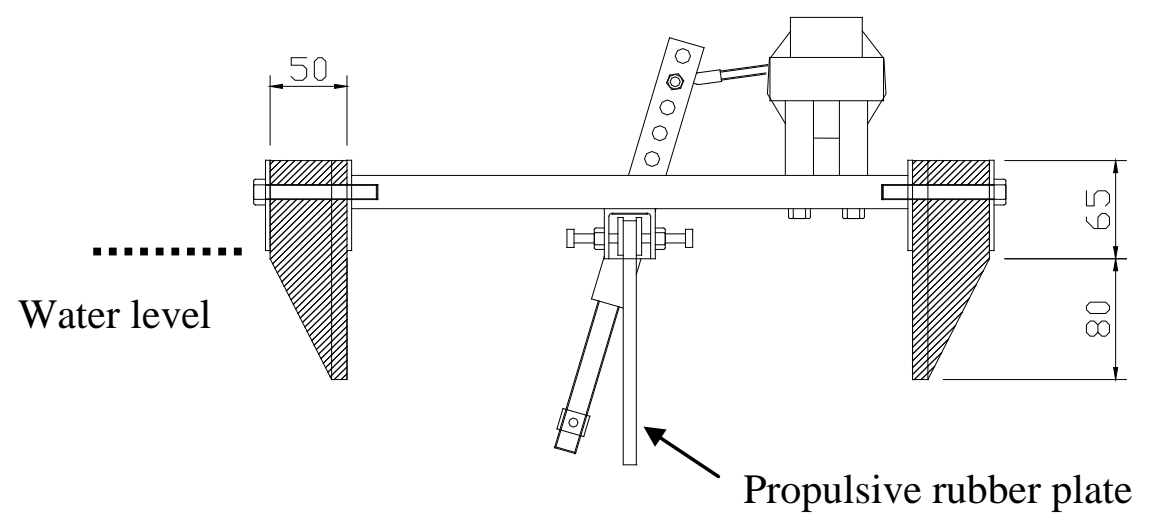

Fig. 4. Design of the catamaran with the wave propulsive plate: dimensions of the pontoons (shaded parts) are given in mm; the propulsive plate is shown detached from the mechanical arm. 


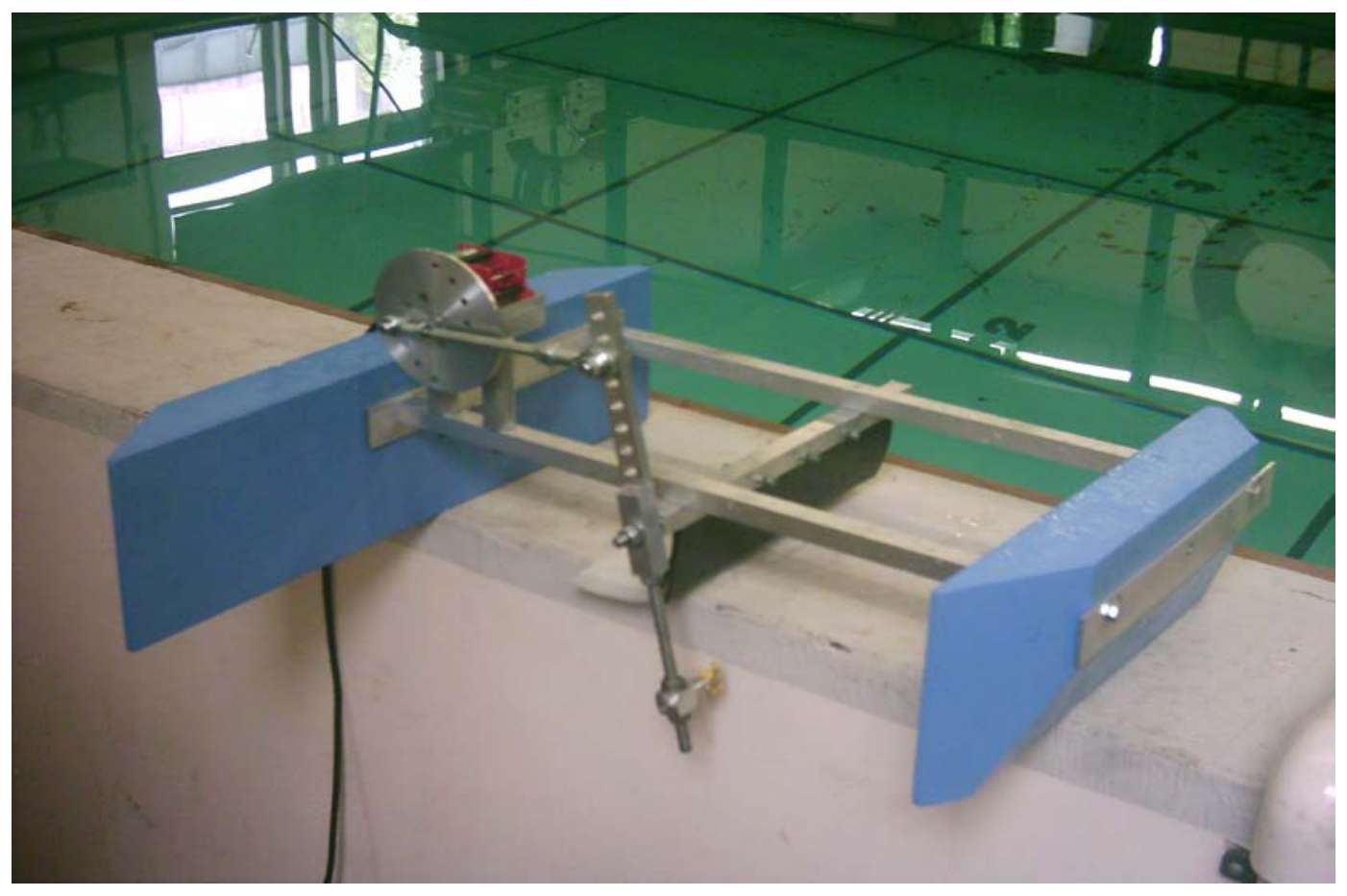

Fig. 5. View of the assembled catamaran with wedge wave propulsive system before its testing in open water (the mechanical arm is detached from the propulsive rubber plate). 


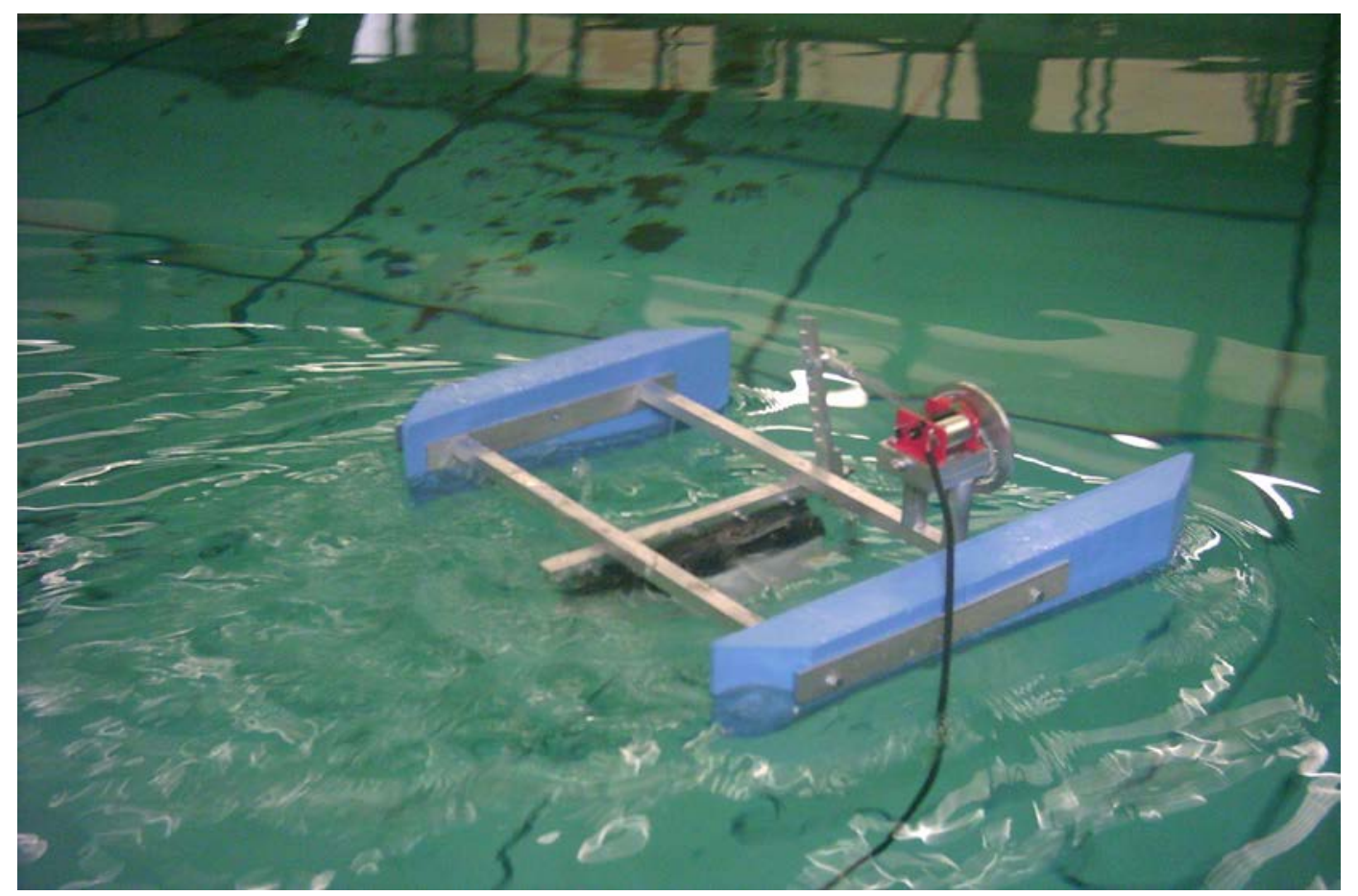

Fig. 6. View of the moving catamaran with guided elastic wave propulsive system during the testing in open water. 